\title{
MODELLING AND CHARACTERISATION OF AN ORTHO-PLANAR MICRO-VALVE
}

\author{
Olivier Smal ${ }^{1}$, Bruno Dehez ${ }^{1}$, Benoit Raucent ${ }^{1}$, Michaël De Volder ${ }^{2}$, Jan \\ Peirs $^{2}$, Dominiek Reynaerts ${ }^{2}$, Frederik Ceyssens ${ }^{3}$, Johan Coosemans ${ }^{3}$, Robert \\ Puers $^{3}$ \\ 'Université Catholique de Louvain, Dept. of Mechanical Engineering, Div. CEREM, Place du \\ levant 2, 1348 Louvain la Neuve, Belgium; ${ }^{2}$ Katholieke Universiteit Leuven, Dept. of \\ Mechanical Engineering, Div. PMA, Celestijnenlaan 300 B, 3001 Leuven, Belgium; \\ ${ }^{3}$ Katholieke Universiteit Leuven, Dept. of Electrical Engineering, Div. MICAS, Kasteelpark \\ Arenberg 10,3001 Leuven, Belgitum.
}

Abstract: The main difficulties encountered in the development of microscale fluidic pumping systems stem from the fact that these systems tend to comprise highly three-dimensional parts, which are incompatible with traditional microproduction technologies. Regardless of the type of pumping principle, most of the hydraulic systems contain valves and in particular a one-way valve. This paper presents the design and modelling of an ortho-planar oneway microvalve. The main advantages of such a valve are that it is very compact and can be made from a single flat piece of material. An analytical model of the spring deflection has been developed and compared to FEM. A prototype with a bore of $1.5 \mathrm{~mm}$ has been build using a micro EDM (electro discharge machining) machine and also tested.

Key words: microvalve; pumping systems; ortho-planar spring.

\section{INTRODUCTION}

Advances in micro-mechanical and in medical areas generate a huge need for microfluidic systems. Recent studies have shown that there is a steadily growing market for Microsystems, and in particular for drug delivery systems. According to 1 the drug delivery market is estimated at US $\$ 20$ billion. Micropumps have a large number of applications: dispensing of 
therapeutic agents into the body, for medical, but also cooling of microelectronic devices, miniature systems for chemical and biological analysis, micropropulsion, etc.

Microfluidic systems might also be used for actuation technologies in microdevices. Recent research revealed that fluidic microactuators might advantageously replace electrostatic and electromagnetic micro-actuators at microscale because they develop higher power and force densities as mentioned in 2 and 3.

Development of Micropumping devices began in the mid 1970's, now leading to many different principles as reported in 4 . Several hundreds of papers reporting new micropumps or analysing micropump operation have been published.

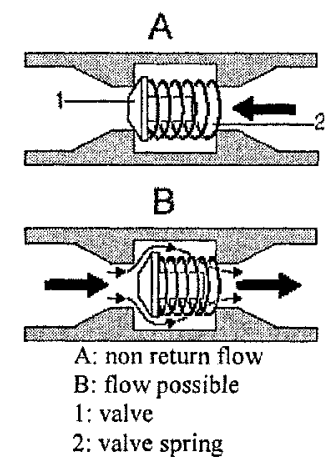

Figure 1. Non return valve principle (courtesy of Bosch)

Regardless of the pumping principle, most micropumps comprise active or passive microvalves. The most common valve is the check valve illustrated in Figure 15. It comes in a wide variety of shapes and materials but always with the same requirements: a low leakage in the reverse direction and a low pressure drop in the inlet flow direction. The spring stiffness should be carefully selected.

The valve presented in figure 1 is however very difficult to produce at micro scale. This paper presents the production and modelling of an orthoplanar one-way microvalve. The major advantage of such a valve is that it can be very compact and simple to build.

The second section focuses on the design principle. The third section describes an analytical model of the spring deflection while section 4 presents an FEM simulation. Section 5 presents the prototype fabrication and section 6 the prototype test and a comparison of models. 


\section{ORTHO-PLANAR MICROVALVE}

Ortho-planar valves are valves based on ortho-planar springs. Such springs which can be either manufactured in or compressed down into a single plane 6. Examples of ortho-planar springs are the Belleville disc spring, the volute spring and the spider spring. Their major advantages are that they are very compact and can be made from a single flat piece of material. In this study, we will concentrate on spider springs, which are by definition manufactured in a plane. Several typical shapes are presented in Figure 2.

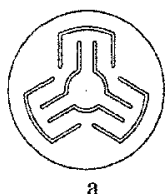

a

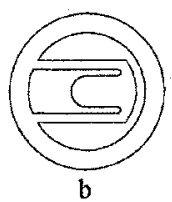

b

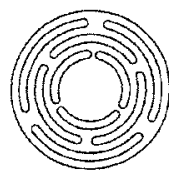

c
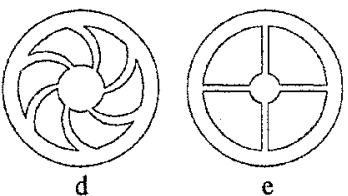

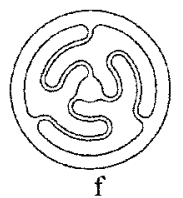

Figure 2. Spiders

The valve principle is presented in Figure 3. The central disc is connected to the outer disc by several beams. When pushing on the central disc in a direction perpendicular to the plate, the beams bend and apply a spring force to the central disc. If the plate is symmetrical then the central disc always stays parallel to the outer disc. Figure 3 shows the valve behaviour in its inflow and back-flow state. When the fluid flows in (case a), the fluid pushes on the central disc and opens the valve. When the inlet pressure drops, the beams, acting like a spring, close the valve (case b). As a consequence, this valve acts as a one-way valve.

The various spiders illustrated in Figure 2 could theoretically be used in this configuration. However practical limitations should be considered: the fluid has to pass trough the spider cuttings. That is why shapes (a), (b) and (c) can be directly rejected because they offer too much resistance to the fluid flow. Spider (d), (e) and (f) are comparable in the principle, but as the beams are longer in $(f)$, the spring stiffness will be smaller and the opening area larger. In the following we will concentrate on that case.

Figure 1 shows that to ensure that the valve is kept closed in its rest position, the spring has to be pre-stressed. This pre-stress defines the opening pressure of the valve. Normally, the opening pressure should be between 0.001 and $0.05 \mathrm{MPa} 7$. When the pressure is high enough, the opening should be as large as possible in order to reduce the pressure drop in the valve. It is therefore very important to have a good model of the spring. 


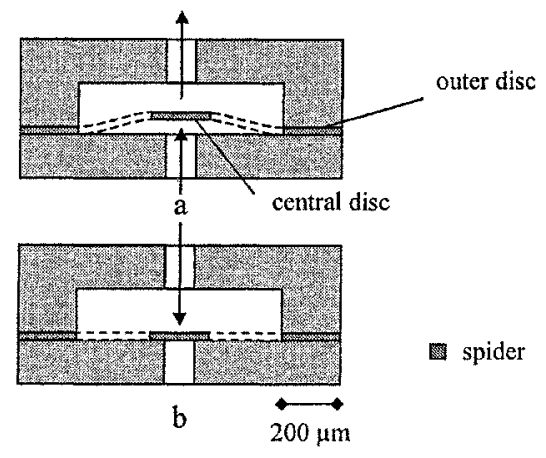

Figure 3. Valve principle

\section{SPRING MODELLING}

Spider have already been modelled and manufactured $[6,8,9]$. However, existing models consider only straight bar spring segments and take into account only the beam's bending, neglecting its torsion. Such a principle is therefore not suitable for curved beams as in case (f) presented in Figure 2. In this section, we propose a new model taking into account bending and torsion of a curved beam.

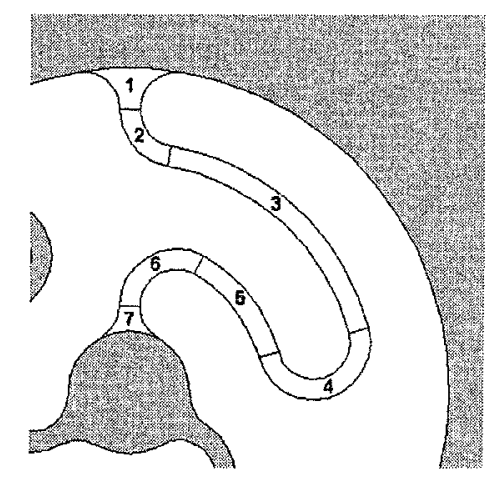

Figure 4. Beam decomposition

The analytical model presented in this section is a deformation model, the fluidic action being modelled by the simple action of the pressure on the central disc. Fluidic action on the beams are neglected 
To model this structure, the valve is considered as a disc suspended on folded beams. The central disc is assumed to be non deformable, all the deformation occuring in the beams. Each beam can be considered as a spring connected in parallel. So the total force applied by the fluid on the central disc is equally divided between each beam The folded beam is divided into successive straight or curved standard elements, see Figure 4 . The first and last element ( 1 and 7$)$ are considered to be straight with constant cross section. Shear forces, moments, displacements and slopes are modelled for the two standard elements using Roark's formulae 10. The directions for forces and slopes are presented in Figure 5.
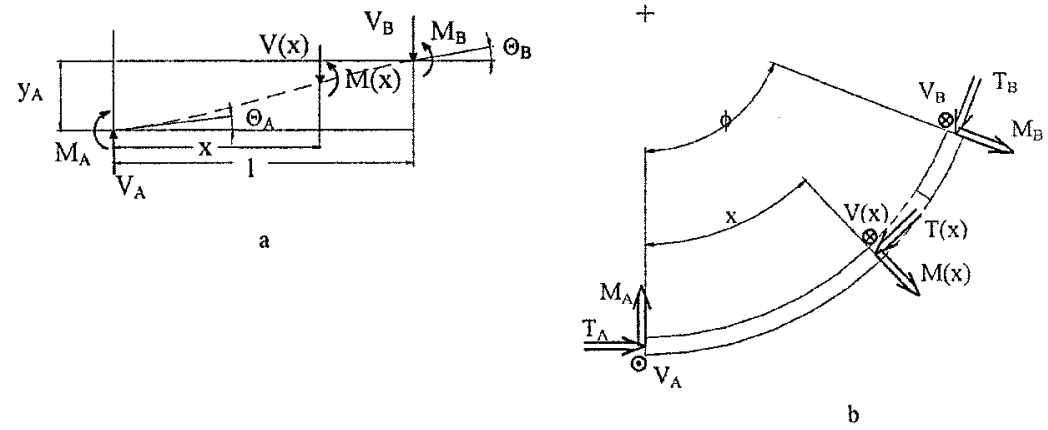

Figure 5. Forces and moment orientation, a: lateral view for a straight beam, b: top view for a curved beam.

Case (b) presents the conventions for elements 2,5 and 6 in Figure 4. Formulas (1) and (2) present the results for this orientation. Formulas for the other orientation (as for elements 3 and 4 in Figure 4) can be easy derived and are not presented here. For a straight bar:

$\left(\begin{array}{c}V(x) \\ M(x) \\ T(x) \\ y(x) \\ \Theta(x) \\ \psi(x)\end{array}\right)=\left(\begin{array}{cccccc}-1 & 0 & 0 & 0 & 0 & 0 \\ x & 1 & 0 & 0 & 0 & 0 \\ 0 & 0 & 1 & 0 & 0 & 0 \\ \frac{x^{3}}{6 E I} & \frac{x^{2}}{2 E I} & 0 & 1 & x & 0 \\ \frac{x^{2}}{2 E I} & \frac{x}{E I} & 0 & 0 & 1 & 0 \\ 0 & 0 & \tan \left(\frac{x T_{A}}{K G}\right) \cdot \frac{1}{T_{A}} & 0 & 0 & 1\end{array}\right) \cdot\left(\begin{array}{c}V_{A} \\ M_{A} \\ T_{A} \\ y_{A} \\ \Theta_{A} \\ \psi_{A}\end{array}\right)$ 
and for a curved beam:

$$
\left(\begin{array}{c}
V(x) \\
M(x) \\
T(x) \\
y(x) \\
\Theta(x) \\
\psi(x)
\end{array}\right)=\left(\begin{array}{cccccc}
1 & 0 & 0 & 0 & 0 & 0 \\
R \sin x & \cos x & -\sin x & 0 & 0 & 0 \\
R(1-\cos x) & \sin x & \cos x & 0 & 0 & 0 \\
C_{1} R^{2} F_{3} & C_{1} R F_{1} & C_{1} R F_{2} & 1 & R \sin x & R(1-\cos x) \\
C_{1} R F_{6} & C_{1} F_{4} & C_{1} F_{5} & 0 & \cos x & \sin x \\
C_{1} R F_{9} & C_{1} F_{7} & C_{1} F_{8} & 0 & -\sin x & \cos x
\end{array}\right) \cdot\left(\begin{array}{c}
V_{A} \\
M_{A} \\
T_{A} \\
y_{A} \\
\Theta_{A} \\
\psi_{A}
\end{array}\right)
$$

with $C_{1}=\frac{R}{E I}$ and where $V$ is the shear force, $M$ the bending moment, $T$ the twisting moment, $\Theta$ the bending slope, $\psi$ the roll slope, $G$ the shear modulus of the material, $K$ a factor depending on the shape and dimensions of the cross section; $F_{1} \ldots F_{9}$ are constant factors (value are given in 10 ).

Boundary conditions at the central disc are:

$$
\begin{aligned}
& V_{\text {disc }}=\frac{F_{\text {fluid }}}{j} \\
& \Theta_{\text {disc }}=0 \\
& \psi_{\text {disc }}=0
\end{aligned}
$$

where $F_{\text {fluid }}$ is the force applied by the fluid, $F_{\text {fluid }}=P \cdot S_{\text {disc }}$

$j$ is the index of beam,

$V_{\text {disc }}$ is the perpendicular force applied by the beam onto the central disc,

$\Theta_{\text {disc }}$ the bending slope on the central disc,

$\psi_{\text {disc }}$ the roll slope on the central disc.

By combining relations (1) and (2), it is possible to evaluate results at any point on the beam starting with the effort at the beam extremity (on the outer disc side) as functions of $M_{N}$ and $T_{N}$ (bending and twisting moments on the central disc). Relation (3) can be used to evaluate $\mathrm{M}_{\mathrm{N}}$ and $\mathrm{T}_{\mathrm{N}}$. 


\section{FEM MODELLING}

force(5) $=5 \mathrm{e}-4$ Subdomain: von Mises stress Subdomain marker: von Mises stress Displacement: Displacement
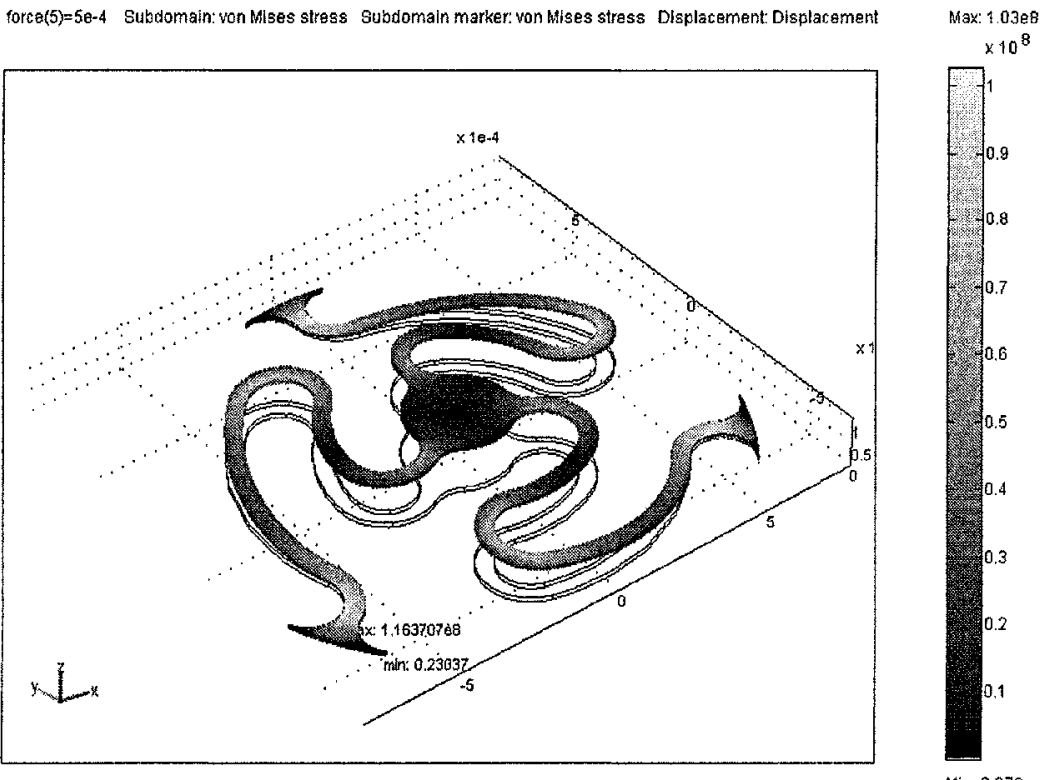

Min: 0.670

Figure 6. FEM simulation of the 3 beams spring

A FEM model has been developed. This is a 3D model, using a drawing of the valve computed with Autodesk Mechanical Desktop $®$. For a given force on the central disc, the values of the displacement and the Von Mises stresses are computed. As for the analytical model, this is only a deformation model, the fluidic part of the valve is not taken into account.

\section{MANUFACTURING}

This part presents only the manufacturing of the spring itself, the other components of the valve will be considered later. This small part is realised using a micro EDM (electro discharge machining) machine. Starting from a stainless steel micro-foil (thickness $=10 \mu \mathrm{m}$ ), the cuttings are made with a standard $150 \mu \mathrm{m}$ diameter electrode. 


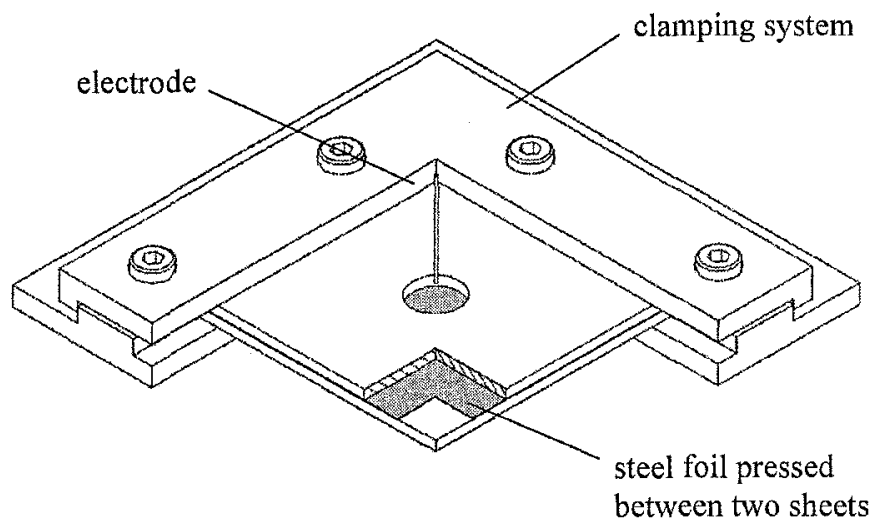

Figure 7. Clamping system

The thin foil is clamped between two thicker stainless steel sheets as illustrated in Figure 7. The upper sheet has a hole to let the electrode work.

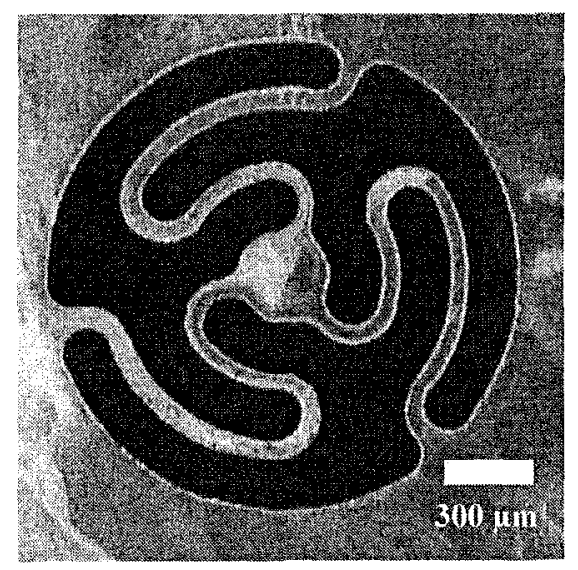

Figure 8. Valve spring

It will be possible to produce a smaller valve design if we work with a thinner electrode (up to $50 \mu \mathrm{m}$ ). On our EDM machine, there is a wire system to reduce the diameter of the electrode before machining. But for a first experiment we prefer to use the standard electrode without any modifications. Figure 8 shows the valve spring prototype. 


\section{RESULTS}

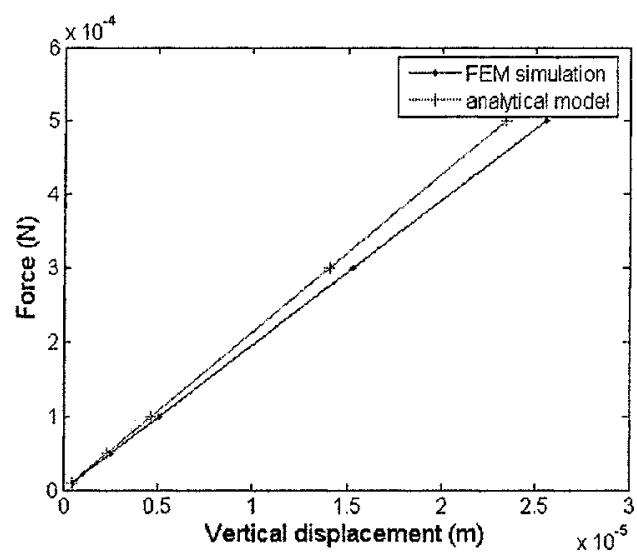

Figure 9. Spring force on the central disc

Figure 9 presents the evolution of the spring force as a function of the vertical displacement of the central disc for a 3-beam valve depicted in Figure 11. Good linearity can be observed. The FEM simulation was also conducted for the various shapes of Figure 11. The results for the stiffness are presented in Table 1 where they are compared with the calculations from the analytical model. The stiffnesses are evaluated with the values of the displacement and the applied force. The approximate model considers the beams as straight bars in the same way as models developed in 8. More precisely, for the case of Figure 4, if $L_{3}$ and $L_{5}$ are the lengths of segment 3 and 5 , the stiffness is computed as being that of a straight beam of length $\mathrm{L}_{3}$ $+L_{5}$ with one end fixed and the other end guided. This explains why, in general, the approximate stiffness is larger than that computed via an analytical model.

Table 1. Stiffnesses for the various valve designs (in $\mathrm{N} \mathrm{m-1)}\left({ }^{*}\right)=$ straight beam

\begin{tabular}{|lccccc|}
\hline Calculations & Valve $^{\circ} 1$ & Valve $^{\circ} 2$ & Valve $^{\circ} 3$ & Valve $^{\circ} 4$ & Valve ${ }^{\circ} 5$ \\
\hline Approximate model & 2.30 & 6.04 & 32.57 & 379.57 & 144.96 \\
Analytical model & 4.32 & 4.62 & 21.38 & 60.17 & 144.96 \\
FEM & 4.36 & 4.76 & 19.59 & 53.08 & 144.85 \\
\hline
\end{tabular}

For valve 5 , the 3 models yield similar results. For a curved beam however, the approximate model produces results between $50 \%$ and 5 times removed from the FEM. The very large difference observed for valve $n^{\circ} 4$ is due to the fact that in this geometry, the lengths of segments 3 and 5 (see Figure 4) are very small, so the corresponding straight bar is extremely short, 
which produces a very high stiffness (the length is powered to three in the formulas). Table 2 shows the proportion of the considered length with respect to the total bar length. On the other hand, our analytical model gives a good approximation, with an error of 1 to $15 \%$. It will therefore be used to evaluate the displacement of the central disc corresponding to the desired pre-stress of the spring.

Table 2. models analysis

\begin{tabular}{|lccccc|}
\hline Calculations & Valve $^{\circ} 1$ & Valve $^{\circ} 2$ & Valve $^{\circ} 3$ & Valve $^{\circ} 4$ & Valve $\mathrm{n}^{\circ} 5$ \\
\hline$\left(\mathrm{L}_{3}+\mathrm{L}_{5}\right) / \mathrm{L} \mathrm{L}_{\text {oral }}$ & 0.69 & 0.61 & 0.51 & 0.34 & 1 \\
\hline
\end{tabular}

The small differences that appear could be explained by the main hypotheses made in our analytical model. The first one is the approximation made on the first and last segment. The shape is assumed to be a simple rectangular beam with constant cross section instead of something somewhat more complex as illustrated in Figure 10.

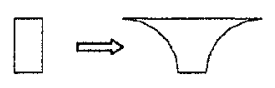

Figure 10. Shape approximation

The second one is the fact that we consider that the entire deformation occurs in the arms. In reality and in the FEM simulation, there is also a deformation in the central disc. The third one comes from the boundary conditions on our beam (see (3)). This hypothesis is a good approximation of what occurs in reality but not the exact truth.

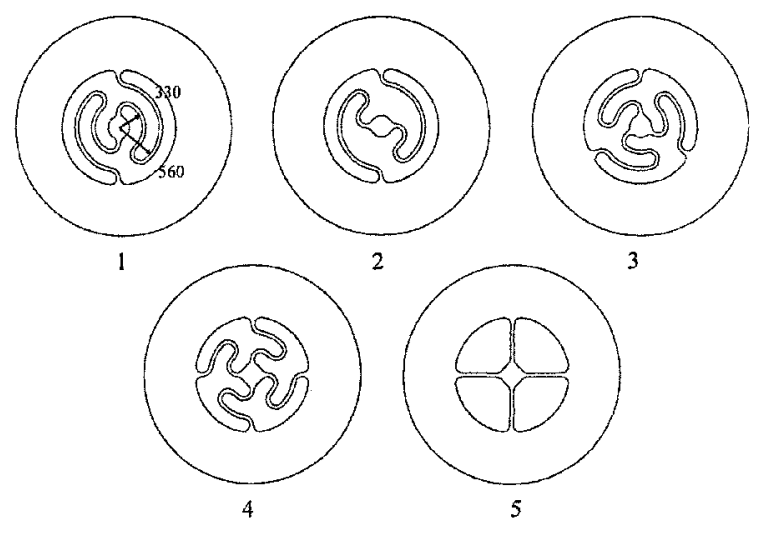

Figure 11. Thickness of the sheet $=10 \mu \mathrm{m}$; central disc diameter $=300 \mu \mathrm{m}$; beam width $=50$ $\mu \mathrm{m} ;$ spring diameter $=1.58 \mathrm{~mm}$ 
Finally the stiffness of the spring presented in Figure 8 is measured using a very accurate microscope equipped with a probe. This probe allows to impose a vertical force from 10 up to $200 \mu \mathrm{N}$ and to measure the induced displacement. Figure 12 presents the results of this test. It is important to note that the slope of the line (linear fit of the data) is quite close to the stiffness calculated and computed via FEM. We find $19.48 \mathrm{Nm}^{-1}$ with the measurements, $19.59 \mathrm{Nm}^{-1}$ with FEM and $21.38 \mathrm{Nm}^{-1}$ by calculations.

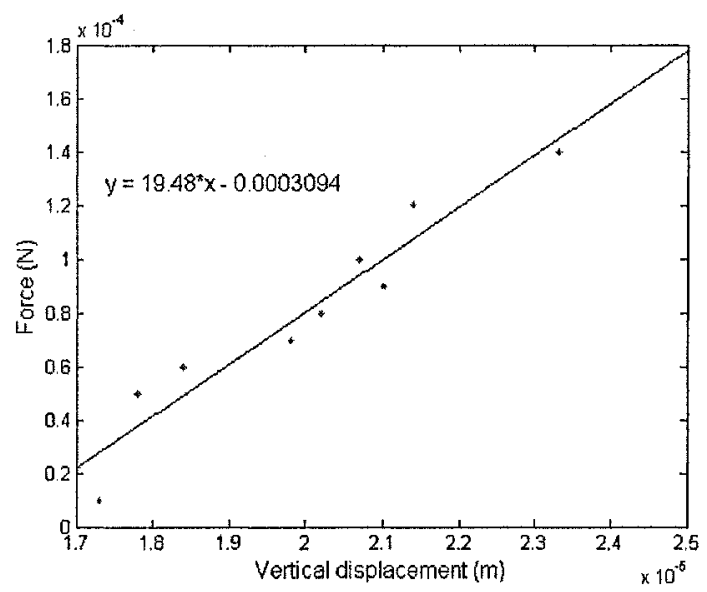

Figure 12. Stiffness measurement on spider $n^{\circ} 3$

In the near future, we will apply the same testing procedure with other spiders to validate our model for the various shapes.

\section{CONCLUSION AND PERSPECTIVES}

The analytical model proposed in this paper proved to be accurate enough for our application. In the near future we will use this model to optimize the valve design. Then we will perform an experimental comparison. Finally we will introduce fluid flow effect on the valve and build a prototype of the complete valve for hydraulic testing. 


\section{BIBLIOGRAPHY}

1. Medical market for Microsystems, J. Malcolm Wilkinson, MSTnews n4 4,2002.

2. Production and Characterisation of a Hydraulic Microactuator, M. De Volder, J. Peirs , D. Reynaerts, J. Coosemans, R. Puers, O. Smal, B. Raucent, Journal of Micromechanics and Microengineering, to appear.

3. A novel hydraulic microactuator sealed by surface tension, M. De Volder, J. Peirs, D. Reynaerts, J. Coosemans, Robert Puers, Olivier Smal, Benoit Raucent, Sensor and Actuation, to appear.

4. A review of Micropumps, D.J. Laser and J.G. Santiago, Journal of Micromechanics and Microengineering 14 (2004) R35-R64.

5. Encyclopedia of technical terms, Bosch, www.ewbc.de.

6. Ortho-planar linear-motion springs, J.J. Parise, L.L. Howell, S.P. Magleby, Mechanism and Machine Theory 36 (2001) 1281-1299.

7. Festo, www3.festo.com

8. Micro check valves for integration into polymeric microfluidic devices, N.T. Nguyen, T.Q. Truong, K.K. Wong, S.S. Ho, C.L.N Low, Journal of Micromechanics and Microengineering 14 (2004) 69-75.

9. Development of large flow rate, robust, passive micro check valves for compact piezoelectrically actuated pumps, B. Li, Q. Chen, D.G. Lee, J. Woolman, G.P. Carman, Sensors and Actuators A 117 (2005) 325-330.

10. Roark's Formulas for Stress \& Strain, 6th edition, W.C. Young, Mc Graw-Hill 1989. 